\title{
Endurance and Performance of Two Different Concepts for Left Ventricular Stimulation with Bipolar Epicardial Leads in Long-Term Follow-Up*
}

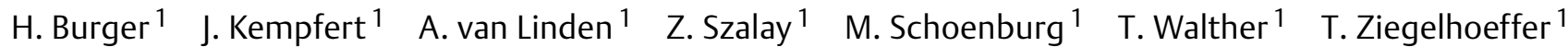 \\ ${ }^{1}$ Department of Cardiac Surgery, Kerckhoff-Klinik, Bad Nauheim, \\ Germany \\ Address for correspondence and reprint requests Dr. Tibor \\ Ziegelhoeffer, M.D., Ph.D., Department of Cardiac Surgery, Kerckhoff- \\ Klinik, Benekestr. 2-8, 61231 Bad Nauheim, Germany \\ Thorac Cardiovasc Surg 2012;60:70-77. \\ (e-mail: tibor.ziegelhoeffer@mpi-bn.mpg.de).
}

\author{
Abstract \\ Keywords \\ - pacing (incl. \\ resynchronization/ \\ biventricular) \\ - electrophysiology \\ (includes mapping) \\ - arrhythmia therapy \\ (includes Maze; \\ surgical ablation) \\ - epicardial leads \\ - cardiac \\ resynchronization \\ therapy \\ - surgical access \\ - screw-in leads \\ - suture-on leads
}

Background Epicardial left ventricular (LV) leads represent an alternative for CRT therapy if transvenous lead implantation fails. Data on endurance, performance, the impact of the surgical approach (lateral minithoracotomy vs. median sternotomy simultaneously with other cardiac surgery), and the optimal technical concept (screw-in vs. suture-on) is limited.

Methods Over a period of 48 months we evaluated 130 consecutive patients with comparable characteristics. A total of 54 screw-in (MyoDex ${ }^{\mathrm{TM}} 1084 \mathrm{~T}, \mathrm{SJM}$ ) and 76 sutureon (Capture Epi 4968, Medtronic) bipolar epicardial steroid-eluting LV leads were implanted either via a left lateral or a median thoracotomy. Sensing, pacing threshold, impedance and NYHA class were recorded at defined time points.

Results No surgery-related death or major complication was observed. At the time of implantation, the pacing threshold, sensing and NYHA class did not differ significantly between the two groups. The impedances of screw-in leads were significantly lower compared to those of suture-on leads. Suture-on leads showed a moderate initial drop in their pacing threshold but afterwards remained stable. Screw-in leads were characterized by a moderate but significant increase in the pacing threshold in the first year followed by a continuous decrease thereafter. Twenty-four months post-implantation no differences between both lead types could be detected. Sensing and NYHA class improved in both groups. The surgical approach had no significant impact on lead functionality.

Conclusion Our study showed that the implantation of epicardial leads was safe with very low complication rates. There was no superior technical epicardial lead concept (screw-in vs. suture-on leads) and all epicardial leads demonstrated an excellent longterm performance and durability. Therefore, it seems that epicardial leads represent a good alternative to transvenous leads and surgeons should be encouraged to implant epicardial leads during concomitant cardiac surgery when the indications for CRT are present. received

January 17, 2011

accepted after revision

May 19, 2011

published online

July 25, 2011

\footnotetext{
* This paper was presented at the annual meeting of the German Society for Thoracic and Cardiovascular Surgery, Stuttgart, February 2010.
} 


\section{Introduction}

Implanted cardiac resynchronization therapy (CRT) systems represent a "gold standard" in the treatment of advanced heart failure if pharmacological therapy (e.g., ACE-blocker, $\beta$ blocker, Spironolacton) alone is not sufficient. Numerous studies have shown that biventricular pacing is very likely to lead to an improvement in left ventricular systolic function in patients with advanced heart failure. The criteria for CRT system implantation are NYHA class III-IV, severely reduced left ventricular systolic function with an ejection fraction (EF) $<35 \%$, and intraventricular conduction delay of QRS $>120 \mathrm{~ms}$. The rationale for CRT is the resynchronization of ventricular dyssynchrony and therefore an improvement in the impaired pump function of the failing ventricles. Moreover, a reverse remodeling of the ventricle may occur. ${ }^{1-8}$

With advances in techniques for retrograde coronary sinus cannulation, a fully transvenous approach has become the technique of choice for CRT. Nowadays, almost all primary LV lead implantations are performed using a transvenous approach. Unfortunately, with the continuously increasing number of patients who have already received a CRT system or are scheduled for CRT implantation, the number of unsuccessful or impossible transvenous LV lead implantations is rising. Moreover, in cases with endocarditis and vegetations on transvenous leads open chest surgery is often necessary to explant the transvenous leads and implant epicardial leads.

Epicardial leads possess several disadvantages compared to transvenous LV leads. Their implantation requires a thoracotomy, mostly a lateral thoracotomy, with all the associated risks of general anesthesia; it can be difficult to fix the lead on the surface of the beating heart; epicardial fat may obscure underlying structures and increase the LV pacing threshold; pericardial adhesions may make access difficult and risky; finally, they are believed to have a high lead failure rate. ${ }^{9,10}$ Unless it is planned as a stand-alone procedure, if CRT is indicated, epicardial leads could be simultaneously im- planted when performing open chest cardiac interventions via a median sternotomy (e.g., CABG, MVR, etc.). ${ }^{11,12}$

Currently, two different technical epicardial lead concepts are available: screw-in and suture-on leads. Despite the fact that both possess theoretical advantages and disadvantages, no comparison between these two epicardial lead types can be found in the current literature. Similarly, data on the endurance and performance of epicardial leads in adults and the impact of the surgical approach is still limited, with only a few studies on a small number of patients available. ${ }^{13}$ Therefore, the aim of our study was to address these issues by retrospectively evaluating 130 patients who underwent epicardial lead implantation.

\section{Methods}

We retrospectively evaluated 130 consecutive patients who underwent implantation of epicardial left ventricular leads between November 2003 and December 2009 in our center. Detailed patient data are summarized in - Table 1.

At the time of implantation, the surgical approach (median or lateral minithoracotomy), the use of cardiopulmonary bypass if necessary, the implanted lead type (screw-in lead MyoDex $^{\mathrm{TM}} 1084 \mathrm{~T}$, St. Jude Medical, St. Paul, MN, USA; sutureon lead Capture Epi 4968, Medtronic, Minneapolis, MN, USA), electrotechnical implantation parameters (sensing [mV], pacing threshold $[\mathrm{V} / 0,5 \mathrm{~ms}]$, impedance $[\mathrm{Ohm}])$, echocardiography data and clinical NYHA class were noted. All parameters were assessed again at 3 months, and every 6 months thereafter. Follow-up was 48 months.

Median sternotomies were performed in the standard manner used for most cardiac surgery procedures (CABG, aortic valve replacement/reconstruction, etc.). Lead implantations via a lateral minithoracotomy were performed under general anesthesia and on the beating heart as previously described. ${ }^{14}$ Briefly, all patients had standard monitoring (ECG, pulse oximetry and invasive arterial monitoring). Right

Table 1 Preoperative patient characteristics.

\begin{tabular}{|c|c|c|c|c|c|}
\hline Lead type & Screw-in $(n=54)$ & Suture-on $(n=76)$ & & Screw-in $(n=54)$ & Suture-on $(n=76)$ \\
\hline Male (n) & 36 & 54 & Diabetes mellitus (n) & 18 & 21 \\
\hline Female $(n)$ & 18 & 22 & Creatinine $(\mathrm{mg} / \mathrm{dl})$ & 1.4 & 1.5 \\
\hline Age (year) & 65.97 & 63.6 & GFR (ml/min) & 48 & 44.1 \\
\hline Height (cm) & 171.5 & 171.4 & $\mathrm{EF}(\%)$ & 24.5 & 22.6 \\
\hline Weight $(\mathrm{kg})$ & 85.4 & 78.4 & NYHA class (n) & 3 & 2.8 \\
\hline BMI $\left(\mathrm{kg} / \mathrm{m}^{2}\right)$ & 28.8 & 26.7 & Sinus rhythm (n) & 32 & 43 \\
\hline ICM (n) & 16 & 27 & Atrial fibrillation (n) & 7 & 20 \\
\hline $\operatorname{DCM}(\mathrm{n})$ & 28 & 32 & Asystolia (n) & 15 & 13 \\
\hline Lead infection (n) & 9 & 8 & Left bundle block (n) & 42 & 56 \\
\hline Hypertension (n) & 34 & 41 & Right bundle block (n) & 2 & 1 \\
\hline CAD (n) & 17 & 30 & QRS Time (ms) & 160.1 & 163.6 \\
\hline
\end{tabular}

BMI: body mass index; CAD: coronary artery disease; DCM: dilative cardiomyopathy; EF: ejection fraction; GFR: glomerular filtration rate; ICM: ischemic cardiomyopathy: QRS: QRS complex 
atrial and right ventricular leads were placed transvenously. Access to the pericardium was achieved by a 5-cm left lateral, midaxillary minithoracotomy in the fourth or fifth intercostal space. The pericardium was exposed and opened anterior to the phrenic nerve. Afterwards screw-in (MyoDex ${ }^{\mathrm{TM}} 1084 \mathrm{~T}$, St. Jude Medical) or suture-on (Capture Epi 4968, Medtronic) leads were placed on the appropriate site. If necessary, the leads were repositioned to guarantee satisfactory parameters.

Results are expressed as mean \pm SEM. The $t$-test (impedance and sensing) and the Mann-Whitney-Wilcoxon test (pacing threshold) were used for statistical analysis.

\section{Results}

\section{Patients and complications}

Between November 2003 and December 2009 we implanted 130 bipolar steroid-eluting epicardial leads. Preoperative patient characteristics are summarized in -Table 1. There were no statistical differences in preoperative characteristics between groups. Fifty-four leads were screw-in (MyoDex ${ }^{\text {TM }}$ 1084T, St. Jude Medical) leads and 76 were suture-on (Capture Epi 4968, Medtronic) leads. In 93 patients a left lateral minithoracotomy was performed, and 37 patients underwent a median sternotomy. Cardiopulmonary bypass was used in 34 patients because of concomitant surgical procedures (CABG, MVR).

Overall, no surgery-related, perioperative or postoperative lead-associated deaths occurred. One patient received epicardial leads because of fulminant endocarditis with severe heart failure and low-cardiac output. This resulted temporarily in an increase in the ejection fraction. Unfortunately, the patient died 2 weeks later due to progressive heart failure with severe mitral regurgitation. Two leads-one screw-in and one suture-on lead-were unipolarized due to the increased pacing threshold. These leads remained stable during followup. Out of a total of six patients listed for heart transplantation, 3 were transplanted, one patient died and 2 patients showed a substantial improvement after epicardial lead implantation and CRT and could be delisted. Infection after epicardial lead implantation via a lateral minithoracotomy occurred in 1 patient with severe adipositas and diabetes mellitus. This infection was only superficial and was successfully treated within a few days without the need for lead explantation.

\section{Follow-up}

A comparison of the electrotechnical parameters of the screw-in and the suture-on leads irrespective of the surgical approach used showed some lead-specific differences. The initial mean pacing threshold of both lead types was nearly identical ( $1.1 \mathrm{~V} / 0.5 \mathrm{~ms}$ for the suture-on vs. $1.2 \mathrm{~V} / 0.5 \mathrm{~ms}$ for the screw-in leads). Whereas the pacing threshold in the group with the suture-on leads showed only a slight decrease (minimum $0.7 \mathrm{~V}$ ) during the whole observation period, in the group with screw-in leads a moderate but significant increase was noted during the first 3 months (maximum $1.5 \mathrm{~V}$ ). Subsequently a continuous decrease in the pacing threshold was observed. There were no differences between both lead types after 24 months ( $0.9 \mathrm{~V} / 0.5 \mathrm{~ms}$ vs. $1.1 \mathrm{~V} / 0.5 \mathrm{~ms})$ (-Fig. 1).

A comparison of impedances showed a significant difference between both groups, a difference that was observed continuously throughout the whole follow-up. Initially, the mean impedance of screw-in leads (as measured at $5 \mathrm{~V}$ ) was $435 \mathrm{Ohm}$ versus $710 \mathrm{Ohm}$ for suture-on leads $(p<0.001)$. In both groups a slight decrease in impedance values was apparent during the early follow-up period. Impedances remained stable for a period between 3 and 36 months, followed by a gradual increase (-Fig. $\mathbf{2}$ ).

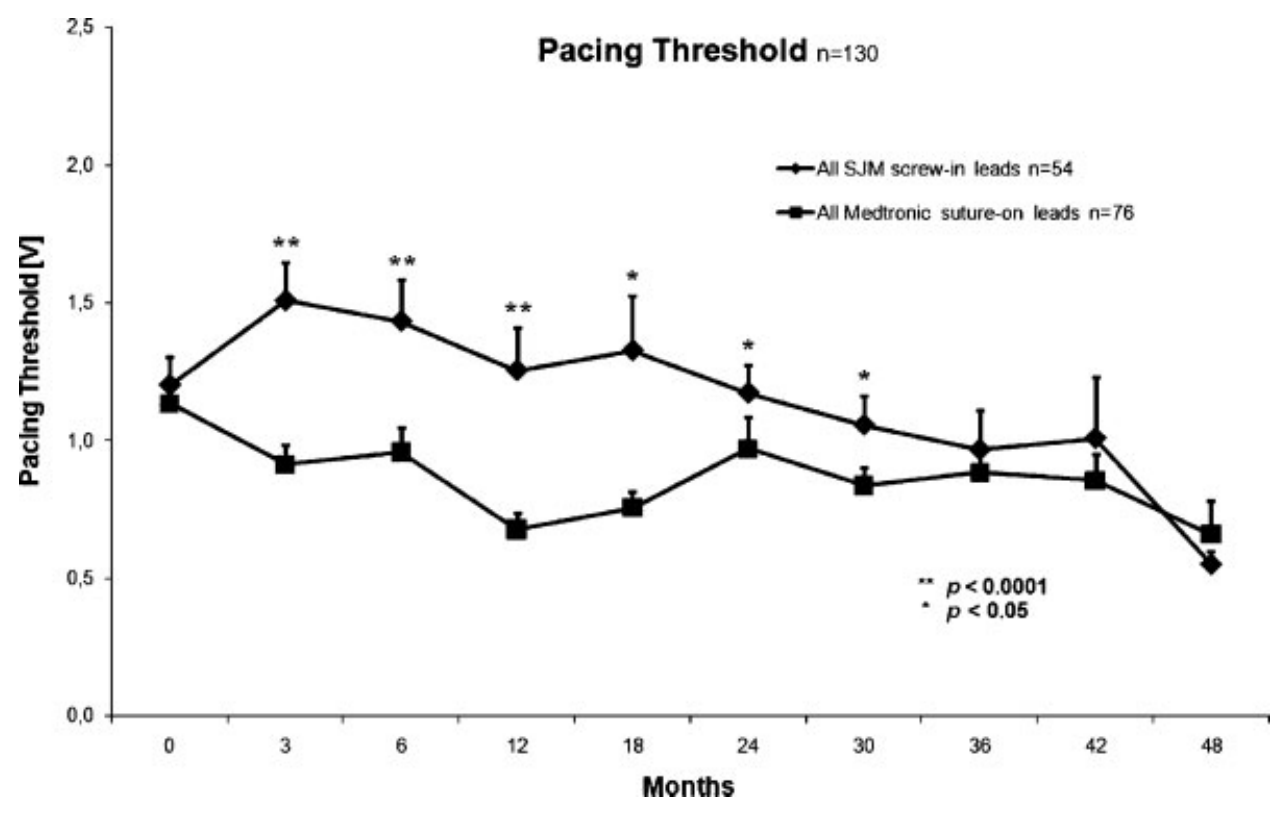

Figure 1 Mean pacing threshold of suture-on vs. screw-in leads. 


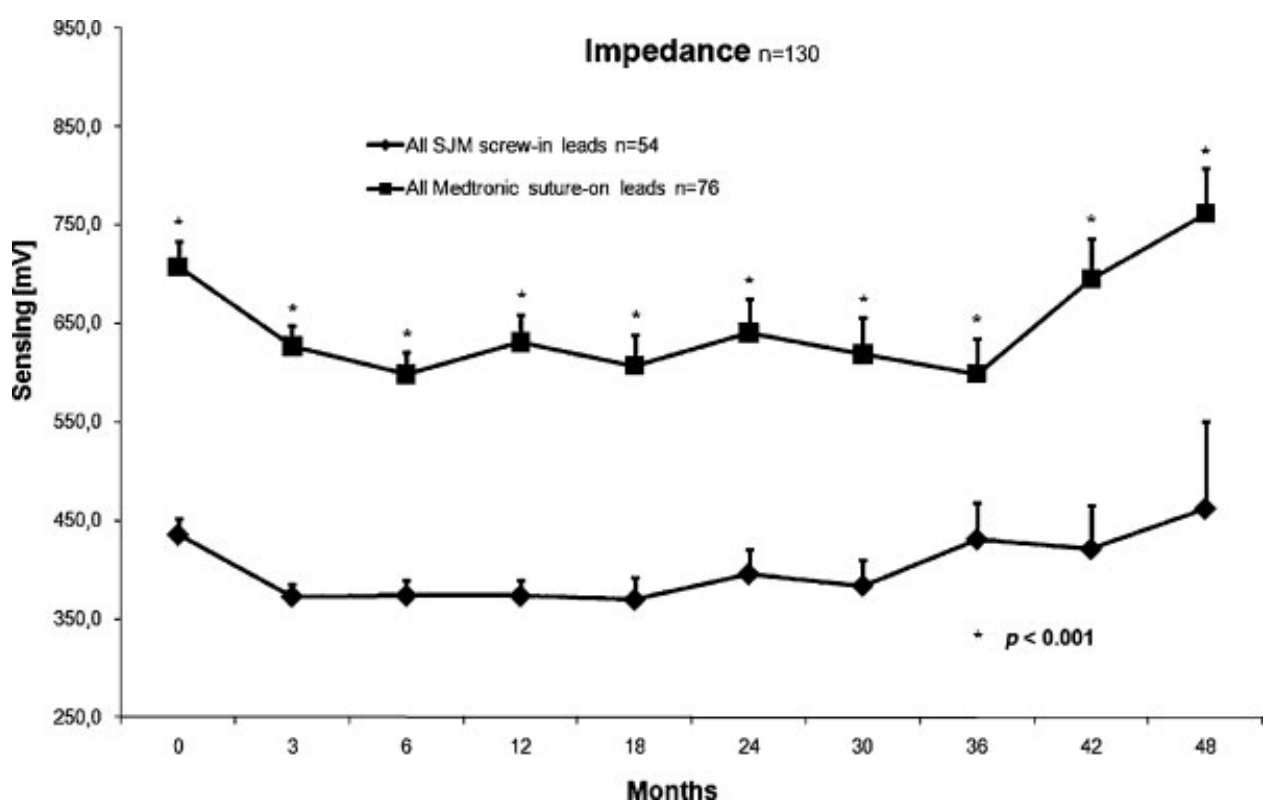

Figure 2 Mean impedance of suture-on vs. screw-in leads.

In contrast to the pacing threshold and impedance, no difference in mean sensing was observed between both lead types. Surprisingly, the already initially good sensing values (10-12 mV) showed an upward trend during the whole follow-up period (-Fig. 3 ).

A subanalysis of electrotechnical parameters was done to investigate whether the surgical approach had an impact on lead performance. Results of the comparison of pacing thresholds, sensing and impedance between both lead types implanted via a lateral minithoracotomy or a median thoracotomy mirrored the data obtained for the comparisons made without taking account of the surgical approach (- Figs. 4 and $\mathbf{5}$ ). However, the results of this analysis must be viewed with caution as only a very low number of screw-in leads $(\mathrm{n}=$ 3) were implanted through a median thoracotomy. Cardio- pulmonary bypass (ECC) was performed in all cases with a median thoracotomy. Patients on cardiopulmonary bypass need to be heparinized and are thus theoretically at a higher risk of bleeding when screw-in leads are used. Such a risk is minimized when suture-on leads are used in this scenario.

Pooled data from both types of lead divided according to the surgical approach differed slightly. These results were largely determined by the lead type used predominantly in the group which underwent a median thoracotomy with cardiopulmonary bypass ( 3 screw-in and 34 suture-on leads) and by the fact that in the group which underwent a lateral minithoracotomy the lead distribution was almost the same (51 screw-in and 42 suture-on leads).

In addition to electrotechnical lead parameters, NYHA class was evaluated preoperatively and during the whole

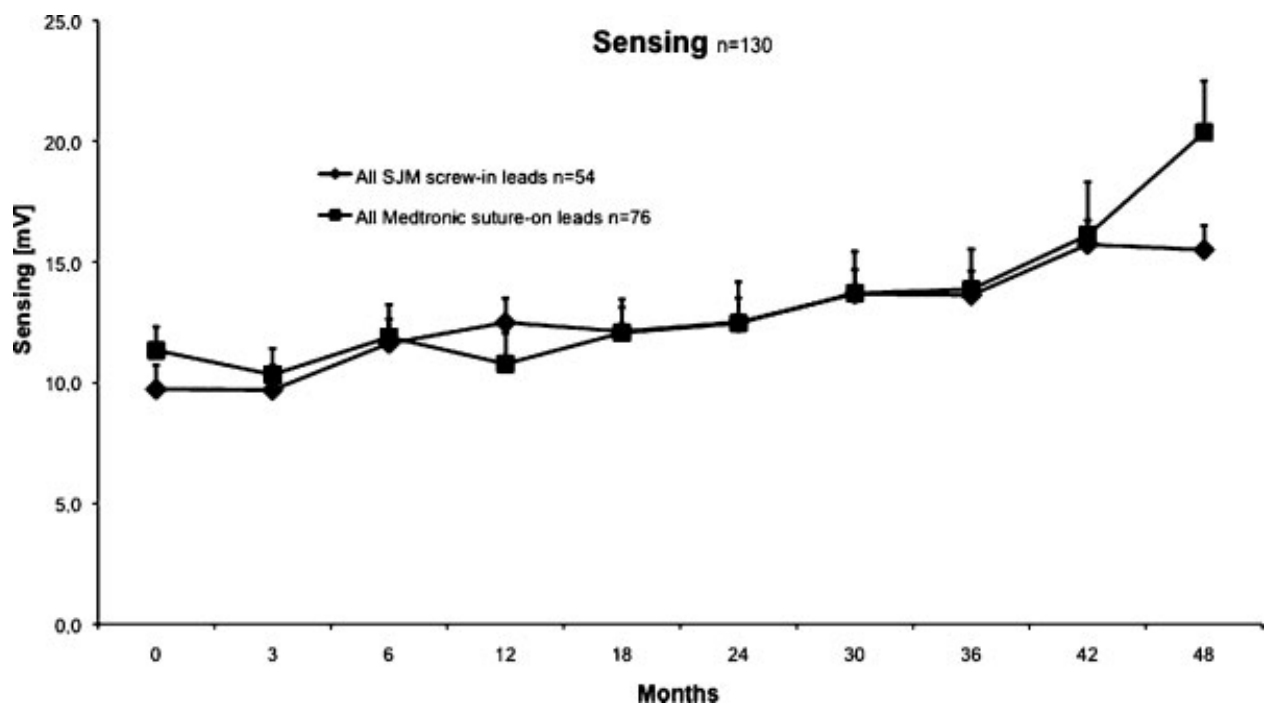

Figure 3 Mean sensing of suture-on vs. screw-in leads. 


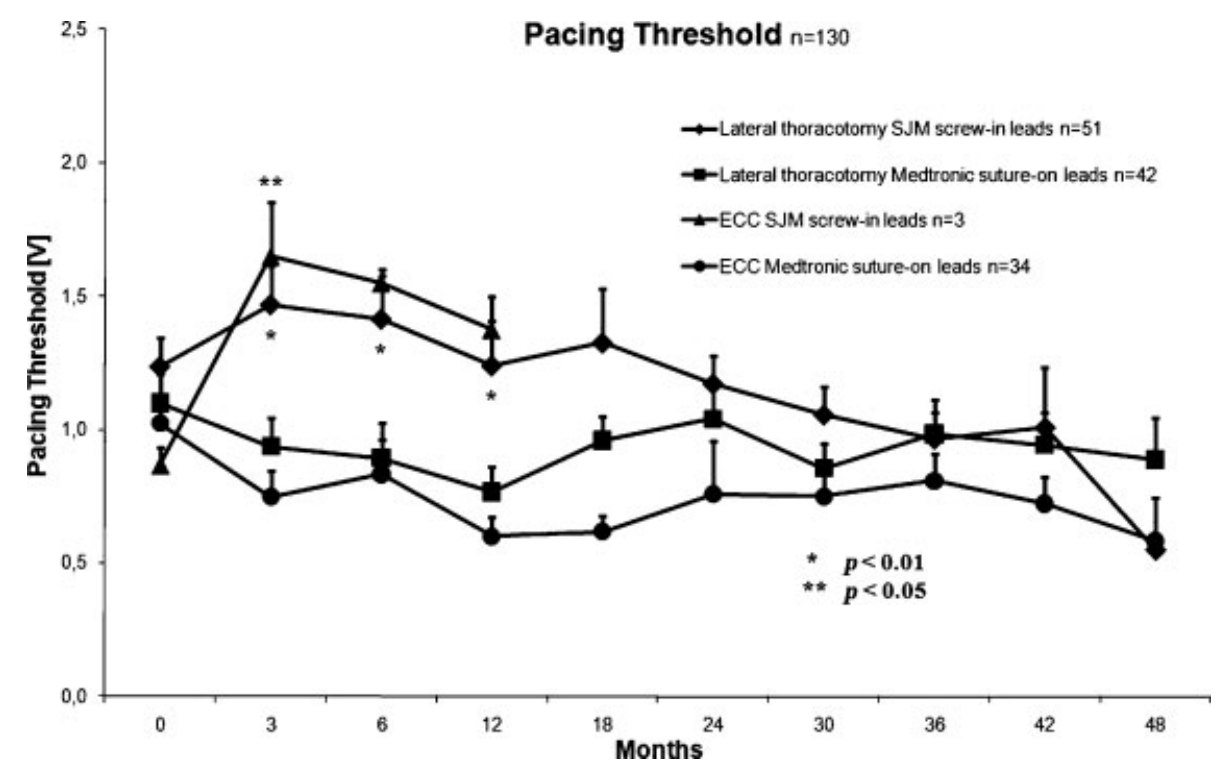

Figure 4 Mean pacing threshold of screw-in and suture-on leads implanted either via lateral minithoracotomy or via median thoracotomy with the use of cardiopulmonary bypass (ECC).

observation period. The distribution of NYHA classes was homogeneous between groups. The mean NYHA class at the time of implantation was almost the same for all groups. Six months after implantation, a nonsignificant decrease in NYHA class was observed in all patients (-Fig. 6). These results confirmed the known and propagated benefit of CRT.

The echocardiography data showed no significant differences between both lead types at any time point. There was a moderate improvement in mitral valve regurgitation, whereas all other measured parameters remained stable over the whole follow-up period of 48 months (-Table 2 ).

\section{Discussion}

In pacing practice, transvenous leads today represent the first choice and the "gold standard" for all types of stimulation: atrial, right ventricular, and left ventricular. In contrast, epicardial leads have been widely used in the past but have been mostly abandoned because of several disadvantages compared to transvenous leads. Epicardial leads are believed to have a high lead failure rate and to be associated with a significant complication rate compared with transvenous leads. ${ }^{9,10,15,17}$ In contrast to this unedifying paradigm in adults, the published data from pediatric

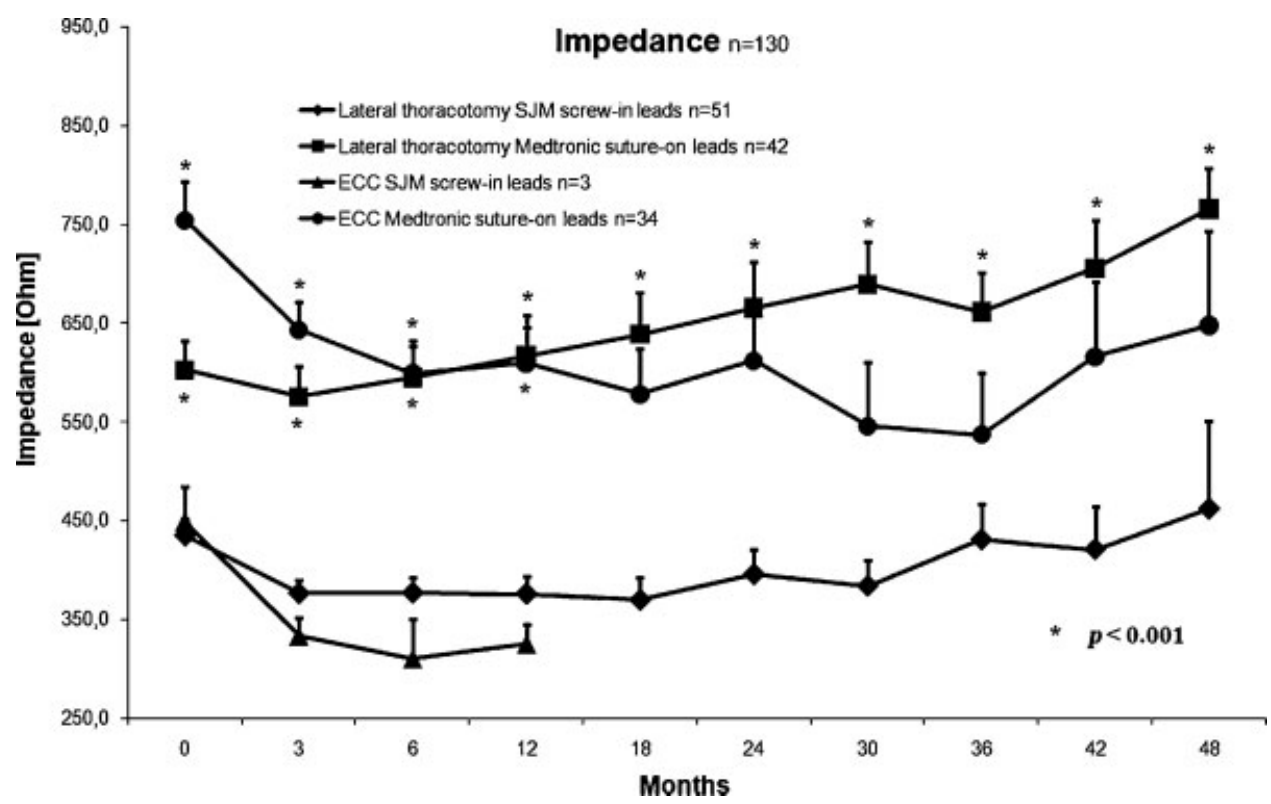

Figure 5 Mean impedances of screw-in and suture-on leads implanted either via lateral minithoracotomy or via median thoracotomy with the use of cardiopulmonary bypass (ECC). 


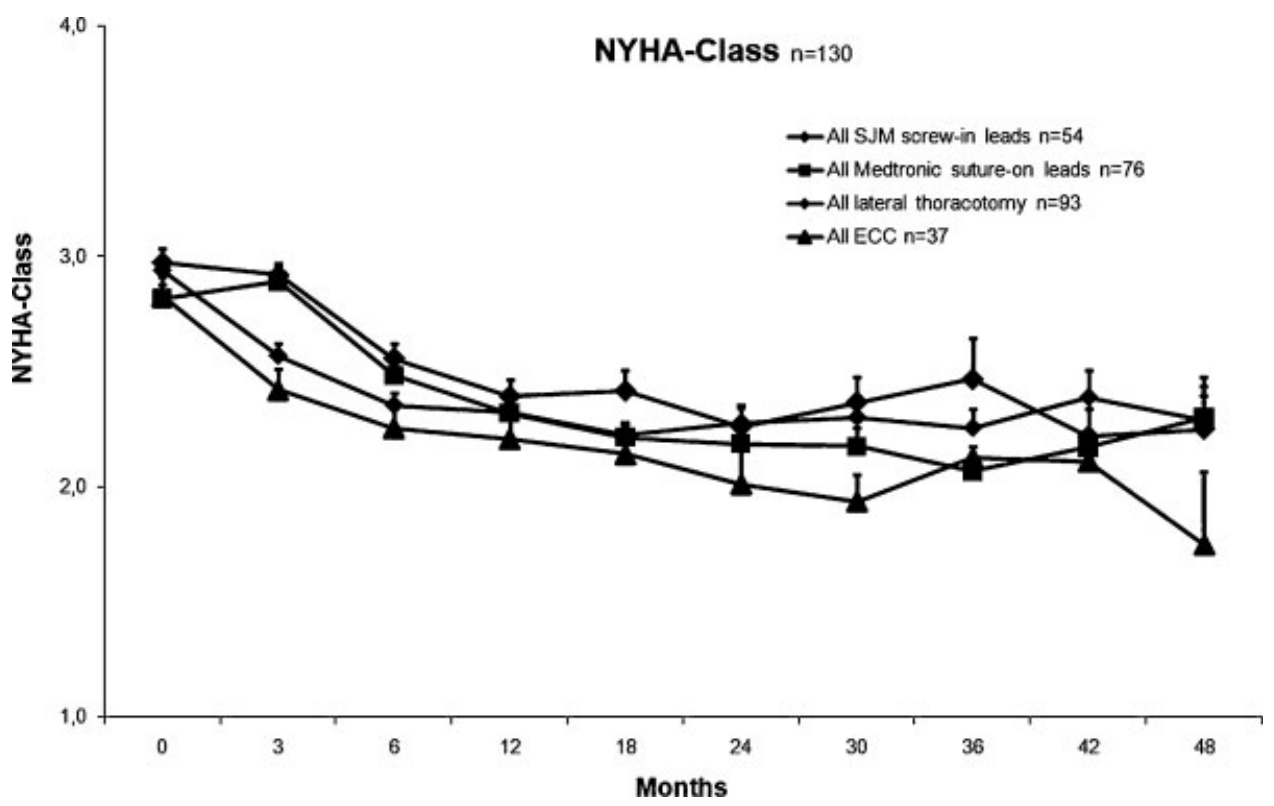

Figure 6 Mean NYHA class according to lead type and surgical approach.

cardiology suggest very good and persistent sensing and pacing threshold characteristics for bipolar steroid-eluting epicardial leads in children with a follow-up of up to 12 years. ${ }^{16}$ This contradiction might be partially due to the differences in the types of patients treated in pediatric cardiology compared to adult cardiology procedures. Pediatric patients are younger and smaller, but they are growing and their underlying pathology differs from that of adults. On the other hand, there are only a few reports with a low number of patients and a limited follow-up period evaluating the long-term performance of "modern" epicardial leads in adults. $10,13,16$

Currently, two different technical epicardial lead concepts are available: screw-in leads and suture-on leads. Despite the fact that both possess theoretical advantages and disadvantages, no comparison between these two epicardial lead types can be found in the current literature.
Both epicardial lead types can be implanted in a standalone procedure (usually via a lateral minithoracotomy) or concomitantly to other cardiac surgery procedures (e.g., CABG, MVR, etc.) via a median thoracotomy. While reports of concomitant epicardial lead implantations during cardiac surgery can be found in the literature, data describing the impact of differing surgical approaches is missing. ${ }^{11,12}$

In our study we retrospectively evaluated all 130 patients who underwent implantation of epicardial left ventricular leads in our center between November 2003 and December 2009. Patients were followed up for up to 48 months. In contrast to the expected pacing threshold and impedance increase, the parameters were excellent and remained stable during the whole observation period. Surprisingly, sensing characteristics even improved.

However, a comparison of the electrotechnical parameters of screw-in and suture-on leads showed some lead-specific

Table 2 Echocardiographic follow-up data.

\begin{tabular}{|l|l|l|l|l|l|}
\hline & Preoperative & $\mathbf{1 2}$ months & $\mathbf{2 4}$ months & 36 months & $\mathbf{4 8}$ months \\
\hline Follow-up (\%) & 100 & 65 & 42 & 29 & 16 \\
\hline EF screw-in lead $(\% \pm$ SEM) & $24.5 \pm 0.92$ & $25.9 \pm 2.01$ & $23.9 \pm 2.49$ & $24.2 \pm 3.93$ & $24.5 \pm 4.25$ \\
\hline EF suture-on lead $(\% \pm$ SEM) & $22.6 \pm 0.83$ & $23.6 \pm 1.15$ & $23.0 \pm 1.27$ & $28.4 \pm 4.55$ & $24.2 \pm 2.18$ \\
\hline Ml screw-in lead (grade \pm SEM) & $1.7 \pm 0.16$ & $1.7 \pm 0.17$ & $1.4 \pm 0.25$ & $0.8 \pm 0.11$ & $1.0 \pm 0.00$ \\
\hline Ml suture-on lead (grade \pm SEM) & $2.1 \pm 0.14$ & $1.2 \pm 0.11$ & $1.5 \pm 0.17$ & $1.4 \pm 0.21$ & $1.2 \pm 0.23$ \\
\hline LVESD screw-in lead $(m m \pm$ SEM) & $57.6 \pm 1.35$ & $56.7 \pm 2.5$ & $60.5 \pm 5.4$ & $53.6 \pm 4.72$ & $57.3 \pm 14.3$ \\
\hline LVESD suture-on lead $(m m \pm$ SEM) & $57.5 \pm 1.63$ & $56.2 \pm 2.25$ & $56.1 \pm 2.85$ & $56.4 \pm 4.72$ & $54.2 \pm 3.62$ \\
\hline LVEDD screw-in lead $(m m \pm$ SEM) & $65.7 \pm 1.09$ & $66.1 \pm 2.15$ & $68.9 \pm 5.24$ & $60.4 \pm 5.45$ & \\
\hline LVEDD suture-on lead (mm \pm SEM) & $66.8 \pm 1.27$ & $64.4 \pm 1.86$ & $67.4 \pm 2.09$ & $66.9 \pm 3.12$ & $64.1 \pm 2.75$ \\
\hline
\end{tabular}

EF: ejection fraction; LVESD: left ventricular end-systolic diameter; LVEDD: left ventricular end-diastolic diameter; MI: mitral valve insufficiency; SEM: standard error of the mean 
differences. Initially nearly identical mean pacing thresholds for both lead types were followed by a slight decrease in the group with suture-on leads whereas in the group with screwin leads a moderate but significant increase was noted during the first 3 months followed by a continuous decrease. After 24 months this difference had disappeared. In contrast, the initial significant difference in impedance between both groups persisted during the whole observation period. No differences between the mean sensing of both lead types were noted.

Screw-in leads possess the advantage compared to suture-on leads that they minimize the invasiveness of the procedure due to the very small lateral minithoracotomy needed. This can be achieved by using a rotatable lead introducer (e.g., EnPath, SJM) for lead positioning and fixation. This advantage is offset by the limited visibility and concomitant higher risk, particularly the higher risk of coronary injury. However, we observed a low complication rate in both groups with epicardial leads. In a recently published study which involved 303 patients who underwent CRT device implantation with implantation of left ventricular leads via a transvenous approach, the incidence of device-related infections was $4.3 \%$. The incidence of endocarditis was $1.3 \%^{18}$. In our patient cohort no surgery-related, perioperative or postoperative lead-associated deaths occurred. No case of endocarditis and only one superficial infection after epicardial lead implantation via lateral minithoracotomy were noted. This infection was successfully treated without the need for lead explantation. This suggests that the implantation of epicardial leads has a much lower complication rate than expected and its complication rate is at least comparable to that associated with the implantation of transvenous leads. Moreover, in contrast to the propagated high failure rate of epicardial leads, in our study only two leads-one screw-in lead and one suture-on lead-were unipolarized due to an increase in the pacing threshold. All other leads remained stable during the whole observation period of 4 years. A subanalysis of electrotechnical parameters excluded the impact of the surgical approach.

Unfortunately, the completeness of follow-up represents the major limitation of our analysis. At 3 months completeness of follow-up was $97 \%$, at six months it was $88 \%$, at 12 months 65\%, at 24 months 42\%. This numbers dropped continuously to $29 \%$ at $36,26 \%$ at 42 months and complete follow-up of 48 months was only achieved in $16 \%$. This was mainly due to the fact that, due to our encouraging results, most implantations were performed in the last two years of the study. Moreover, some patients were lost to follow-up because cardiologists outside our hospital performed the regular system checkups. Nevertheless, the remaining patients showed very constant values in every measure with a very low standard error of the mean. This suggests that despite the reduced number of patients at the end of the observation period, our results can be interpolated as offering a meaningful prediction. Of course, further follow-up in the coming years is needed to finalize and definitely confirm our findings.

\section{Conclusions}

Our study shows that the implantation of epicardial leads is safe with a very low complication rate. Neither of the technical epicardial lead concepts (screw-in vs. suture-on leads) was found to be superior, and all epicardial leads showed an excellent long-term performance and durability. It therefore appears that epicardial leads represent a good alternative to transvenous leads and surgeons should be encouraged to implant epicardial leads during concomitant cardiac surgery if indications for CRT are present.

\section{References}

1 St John Sutton MG, Plappert T, Abraham WT, et al. Effect of cardiac resynchronization therapy on left ventricular size and function in chronic heart failure. Circulation 2003;107;19851990

2 Auricchio A, Stellbrink C, Sack S, et al. Long-term clinical effect of hemodynamically optimized cardiac resynchronization therapy in patients with heart failure and ventricular conduction delay. J Am Coll Cardiol 2002;39;2026-2033

3 Cazeau S, Leclercq C, Lavergne T, et al. Effects of multisite biventricular pacing in patients with heart failure and intraventricular conduction delay. N Engl J Med 2001;344;873-880

4 Abraham WT, Fisher WG, Smith AL, et al. Cardiac resynchronization in chronic heart failure. N Engl J Med 2002;346;18451853

5 Young JB, Abraham WT, Smith AL, et al. Combined cardiac resynchronization and implantable cardioversion defibrillation in advanced chronic heart failure: the MIRACLE ICD Trial. JAMA 2003;289;2685-2694

6 Bristow MR, Saxon LA, Boehmer J, et al. Cardiac-resynchronization therapy with or without an implantable defibrillator in advanced chronic heart failure. N Engl J Med 2004;350;21402150

7 Cleland JG, Daubert JC, Erdmann E, et al. Longer-term effects of cardiac resynchronization therapy on mortality in heart failure [the CArdiac REsynchronization-Heart Failure (CARE-HF) trial extension phase]. Eur Heart J 2006;27;1928-1932

8 Auricchio A, Stellbrink C, Butter C, et al. Clinical efficacy of cardiac resynchronization therapy using left ventricular pacing in heart failure patients stratified by severity of ventricular conduction delay. J Am Coll Cardiol 2003;42;2109-2116

9 Lau EW. Achieving permanent left ventricular pacing-options and choice. PACE 2009;32;1466-1477

10 Hansky B, Vogt J, Gueldner H, et al. Linksventrikuläre Stimulation und CRT: Welche Elektrode passt zu welcher Vene? [Left ventricular pacing and CRT. What CV lead fits into which vein?]. Herzschrittmacherther Elektrophysiol 2006;17;01):I14I19

11 Mizuno T, Tanaka H, Makita S, Tabuchi N, Arai H, Sunamori M. Biventricular pacing with coronary bypass and Dor's ventriculoplasty. Ann Thorac Surg 2003;75(3):998-999

12 Golzio PG, Anselmino M, Comoglio C, Vinci M, Rinaldi M, Trevi GP. Biventricular pacing concomitant to on-pump heart surgery: a case series. J Cardiovasc Surg (Torino) 2009;50(6):801806

13 Mair H, Sachweh J, Meuris B, et al. Surgical epicardial left ventricular lead versus coronary sinus lead placement in biventricular pacing. Eur J Cardiothorac Surg 2005;27;235-242

14 Doll N, Opfermann UT, Rastan AJ, et al. Facilitated minimally invasive left ventricular epicardial lead placement. Ann Thor Surg 2005;79(3):1023-1025 
15 Saggau W, Sack FU, Lange R, et al. Superiority of endocardial versus epicardial implantation of the implantable cardioverter defibrillator (ICD). Eur J Cardiothorac Surg 1992;6(4):195-200

16 Doll N, Piorkowski C, Czesla M, et al. Epicardial versus transvenous left ventricular lead placement in patients receiving cardiac resynchronization therapy: results from a randomized prospective study. Thorac Cardiovasc Surg 2008;56(5):256-261
17 Tomaske M, Gerritse B, Kretsers L, et al. A 12-year experience of bipolar steroid-eluting epicardial pacing leads in children. Ann Thorac Surg 2008;85;1704-1711

18 Romeyer-Bouchard C, Da Costa A, Dauphinot V, et al. Prevalence and risk factors related to infections of cardiac resynchronization therapy devices. Eur Heart J 2010;31(2):203-210 